\title{
Extended Recursive Filtering Estimation of Detector Offset Nonuniformity in Infrared Imaging Systems ${ }^{\star}$
}

\author{
César San-Martin ${ }^{1}$, Jorge Pezoa ${ }^{2}$, Sergio Torres ${ }^{2}$, Pablo Meza ${ }^{1}$, \\ and Diana Gutierrez ${ }^{3}$
}

${ }^{1}$ Dep. of Electrical Eng., University of La Frontera. Casilla 54-D, Temuco, Chile

${ }^{2}$ Dep. of Electrical Eng., University of Concepción. Casilla 160-C, Concepción, Chile

${ }^{3}$ Department of Biomedical Eng., Manuela Beltran University, Bogotá, Colombia

csmarti@ufro.cl, \{jpezoa, sertorre\}@udec.cl, pmeza003@pinhue.ufro.cl

\begin{abstract}
In [1] we presented a recursive filter to estimate the detector offset nonuniformity (NU) noise present in infrared (IR) imaging systems. Such a filter was derived considering an estimation time-window short enough so that the offset NU can be regarded as a constant in noise. Since the offset NU is non-stationary, upon the arrival of new blocks of IR data new estimates of the offset NU have to be computed. In this paper, this recursive filter is extended by adding to the so-called intra-block processing (where offset NU is a constant) an inter-block processing algorithm. The inter-block processing algorithm considers the time varying effect in the offset $\mathrm{NU}$ and models it as a discrete-time Gauss-Markov random process. So, the extended filter is designed to compensate continuously for the NU when new blocks of frames arrive. In addition, the theoretical analysis of the estimator provides us expressions for selecting the appropriate parameters required by the algorithm. The ability of the method to compensate for the offset NU is demonstrated by employing several mid-wavelength IR video sequences.
\end{abstract}

Keywords: Image Sequence Processing, Infrared Focal Plane Arrays, Recursive Filtering, Nonuniformity Correction Method.

\section{Introduction}

Infrared (IR) cameras employ an IR sensor to digitize the information. Due to its high performance, the most employed integrated technology in IR sensors is the Focal Plane Array (FPA). An IR-FPA is a die composed of a group of photodetectors placed in a focal plane forming a matrix of $X \times Y$ pixels, which gives the sensor the ability to collect the IR radiation. The nonuniformity (NU) noise in IR imaging sensors, which is due to pixel-to-pixel variation in the detectors'

\footnotetext{
* This work was partially supported by Universidad de La Frontera, P. DIUFRO DI080048. The authors wish to thank Ernest E. Armstrong (OptiMetrics Inc., USA) for collecting the data, and the United States Air Force Research Laboratory, Ohio, USA.
} 
response, can considerably degrade the quality of IR images since it results in a fixed-pattern noise (FPN) that is superimposed on the true image. Further, what makes the problem worse is that the NU noise varies over time, and depending on the FPA technology employed, this drift can take from minutes to hours. In order to solve this problem, several scene-based nonuniformity correction (NUC) techniques have been developed 234 . Scene-based techniques perform the NUC using only the video sequences that are being imaged, not requiring any kind of laboratory calibration technique. Our group has been active in the development of novel scene-based algorithms for NUC based on statistical estimation theory. In previous works, we have developed a Gauss-Markov model to capture the slow variation in the FPN and such model was used to adaptively estimate the NU within blocks of IR video sequences using different types of Kalman filters [567].

Recently, a recursive filter to estimate the offset NU at each detector using solely scene data has been developed [1]. The method is based on two key assumptions: i) the input irradiance at each detector is a random variable uniformly distributed in a range that is common to all detectors in the FPA; and ii) a block of frames is considered to be short enough so that the offset can be assumed as a random constant in noise. Then, within a block, the offset NU at each detector can be optimally and recursively estimated in the mean-squares sense. Since the NU noise slowly drifts its intensity from one block to another, after the arrival of new blocks of data a new estimate for the offset NU is required. Clearly, new estimates are needed because of the assumption on the lack of drift in the offset NU. In this paper, the aforementioned recursive filter is extended adding to the intra-block processing, where the offset is assumed to be constant, an inter-block processing algorithm that considers the slow and random temporal drift in the offset NU. The extension to the algorithm relies on the following assumptions: i) the input irradiance is a random white process following an uniform distribution in a range that is common to all detectors; ii) within each block of frames the offset NU is assumed to be constant; and iii) the slow and random drift is modeled by a discrete-time Gauss-Makov random process 5678 . The extended filter is designed to continuously compensate for the NU when new blocks of frames arrive, and more importantly, the assumption on the stationarity of the offset NU is relaxed.

This paper is organized as follows. In Section 2 the IR-FPA model and the scene-based NUC method by means of the extended recursive filtering are presented. In Section 3 the main parameters required by the algorithm for its computing implementation are discussed. In Section 4 the extended recursive filtering technique is tested using raw mid-wavelength IR video sequences. In Section 5 the conclusions of the paper are summarized.

\section{Inter-block Recursive Estimation of the Offset}

This work is focused on IR FPAs where the NU noise is mainly generated by spatial and temporal variations in the detectors' offset, in the same way as is stated in 11. Then, we suppose that the gain of the detector is a known 
constant either given by the manufacturer of the camera or calculated by using a priori information of the camera under study. As a consequence of the foregoing assumption, for a given pixel and for a given block of frames short enough to consider the offset NU as a constant, the output response at each photodetector, $y_{k}(n)$, is written as

$$
y_{k}(n)=A_{k} x_{k}(n)+B_{k}+v_{k}(n),
$$

where $n$ is the frame number, $k$ denotes the block number, $A_{k}$ is the gain of the detector in the block $k, x_{k}(n)$ represents the true input irradiance collected by the photodetector during the integration time in the $k$ th block, $B_{k}$ is the offset NU noise in the $k$ th block, and the term $v_{k}(n)$ is the additive temporal noise associated to the photodetector. Remark: The aforementioned model represents any single pixel in the array; consequently, all operations shown in this paper are performed on a pixel-by-pixel basis.

The goal of this paper is to develop an inter-block processing algorithm for the recursive filter presented in [1], which assumes that the offset NU is constant in each block. Following 6 67, the slow and random block-to-block drift in the offset NU is modeled by a Gauss-Markov random process. Mathematically we have

$$
B_{k}=\alpha_{k} B_{k-1}+W_{k},
$$

where $\alpha_{k}$ is a constant modeling the magnitude of the drift from the $(k-1)$ th to $k$ th block and $W_{k}$ is a zero mean white noise process. Note that the NU offset in the $k$ th block is assumed to be constant. Note also that a consequence of $\mathrm{E}\left[\boldsymbol{W}_{k}\right]=0$ is that $\mathrm{E}\left[B_{k}\right]=\left(\prod_{j=1}^{k} \alpha_{j}\right) \mathrm{E}\left[B_{-1}\right]$, for $\mathrm{E}\left[B_{-1}\right] \neq 0$.

Considering that the $k$ th block contains a sequence of $l_{k}$ frames, we propose a linear estimator for recursively estimating the offset $\mathrm{NU}$ in a block-by-block fashion

$$
\hat{B}_{k}=C_{k} \hat{B}_{k-1}+\boldsymbol{Y}_{k}^{T} \boldsymbol{K}_{k},
$$

where $\hat{B}_{k}$ and $\hat{B}_{k-1}$ are the estimates of the offset at the $k$ th and $(k-1)$ th block, respectively, and $T$ represents the vector transpose operation. The coefficients of the estimator are the scalar $C_{k}$ and the column vector $\boldsymbol{K}_{n}=$ $\left[K_{k}(1) K_{k}(2) \ldots K_{k}\left(l_{k}\right)\right]^{T}$, where $\operatorname{dim}\left(\boldsymbol{K}_{n}\right)=l_{k}$.

Let the vectors $\boldsymbol{Y}_{k}, \boldsymbol{x}_{k}$, and $\boldsymbol{V}_{k}$ be the observation, the true input irradiance, and the additive noise vector, respectively, all of them associated to the $k$ th block of data. Let these vectors be defined as: $\boldsymbol{Y}_{k} \triangleq\left[y_{k}(1) y_{k}(2) \ldots y_{k}\left(l_{k}\right)\right]^{T}$, $\boldsymbol{X}_{k} \triangleq\left[\begin{array}{llll}x_{k}(1) & x_{k}(2) & \ldots & x_{k}\left(l_{k}\right)\end{array}\right]^{T}$, and $\boldsymbol{V}_{k} \triangleq\left[\begin{array}{llll}v_{k}(1) & v_{k}(2) \ldots v_{k}\left(l_{k}\right)\end{array}\right]^{T}$. Using sucha a vector notation equation (1) can be recasted as

$$
\boldsymbol{Y}_{k}=A_{k} \boldsymbol{X}_{k}+B_{k} \mathbf{1}+\boldsymbol{V}_{k},
$$

where $\mathbf{1}$ is an all-ones vector of dimension $l_{k}$.

In order to specify the filter coefficients $C_{k}$ and $\boldsymbol{K}_{k}$ we invoke the Orthogonality Principle in the following form

$$
\begin{aligned}
& \text { i) } \mathrm{E}\left[\hat{B}_{k}\right]=\mathrm{E}\left[B_{k}\right], \forall k \\
& \text { ii) } \mathrm{E}\left[\boldsymbol{Y}_{k}\left(B_{k}-\hat{B}_{k}\right)\right]=\mathbf{0}, \forall k
\end{aligned}
$$

where $\mathbf{0}$ is a column null vector of length $l_{k}$. Using (5) we obtain 


$$
C_{k}=\alpha_{k}\left(1-\boldsymbol{\Psi}_{k}^{T} \boldsymbol{K}_{k}\right)
$$

where $\boldsymbol{\Psi}_{k} \triangleq\left(1+A \mu_{X_{k}} \mu_{B_{k}}^{-1}\right) \mathbf{1}=\psi_{k} \mathbf{1}$ with: $\mu_{X_{k}}=\mathrm{E}\left[X_{k}\right], \mu_{B_{k}}=\mathrm{E}\left[B_{k}\right]$, and $\psi_{k}=\left(1+A \mu_{X_{k}} \mu_{B_{k}}^{-1}\right)$. Now, if we expand the terms in (6) and use equations (3) and (7), after some algebraic manipulation it is possible to obtain

$$
\begin{aligned}
& \mathrm{E}\left[\boldsymbol{Y}_{k} B_{k}\right]=\left(A \mu_{X_{k}} \mu_{B_{k}}+\mathrm{E}\left[B_{k} B_{k}\right]\right) \mathbf{1} \\
& \mathrm{E}\left[\boldsymbol{Y}_{k} \hat{B}_{k}\right]=\left(A \mu_{X_{k}} \mu_{B_{k}}+\mathrm{E}\left[B_{k} \hat{B}_{k}\right]\right) \mathbf{1}+\boldsymbol{S} \boldsymbol{K}_{k},
\end{aligned}
$$

where $\boldsymbol{S}=A^{2} \mathrm{E}\left[\boldsymbol{X}_{k} \boldsymbol{X}_{k}^{T}\right]+\sigma_{v}^{2} \boldsymbol{I}_{l_{k} \times l_{k}}-A^{2} \mu_{X_{k}}^{2} \mathbf{1 1}^{T}$ with $\boldsymbol{I}_{l_{k} \times l_{k}}$ being the $l_{k} \times l_{k}$ identity matrix. Hence, applying (6) and noting that the Orthogonality Principle implies that $\sigma_{\varepsilon(k)}^{2}=\mathrm{E}\left[\left(B_{k}-\hat{B}_{k}\right) B_{k}\right]$, a closed-form expression for $\boldsymbol{K}_{k}$ can be finally obtained

$$
\boldsymbol{K}_{k}=\sigma_{\varepsilon(k)}^{2} \boldsymbol{S}^{-1} \mathbf{1}
$$

Finally, in order to complete the specification of the filter coefficients, a recursive expression for $\sigma_{\varepsilon(k)}^{2}$ has to be obtained. Using (2), (3), and (7) it is possible to obtain

$$
\sigma_{\varepsilon(k)}^{2}=\left(1-\mathbf{1}^{T} \boldsymbol{K}_{k}\right) \mathrm{E}\left[B_{k} B_{k}\right]-\alpha_{k}\left(1-\boldsymbol{\Psi}_{k}^{T} \boldsymbol{K}_{k}\right) \mathrm{E}\left[\hat{B}_{k-1} B_{k}\right]-A \mu_{X_{k}} \mu_{B_{k}} \mathbf{1}^{T} \boldsymbol{K}_{k} .
$$

The term $\mathrm{E}\left[\hat{B}_{k-1} B_{k}\right]$ can be obtained plugging equation (2) in $\sigma_{\varepsilon(k-1)}^{2}=\mathrm{E}\left[\left(B_{k-1}-\right.\right.$ $\left.\hat{B}_{k-1}\right) B_{k-1}$ ] yielding

$$
\sigma_{\varepsilon(k-1)}^{2}=\alpha_{k}^{-2}\left(\mathrm{E}\left[B_{k}^{2}\right]+\sigma_{w}^{2}\right)-\alpha_{k}^{-1} \mathrm{E}\left[\hat{B}_{k-1} B_{k}\right]
$$

Solving the last equation for $\mathrm{E}\left[\hat{B}_{k-1} B_{k}\right]$ we obtain

$$
\mathrm{E}\left[\hat{B}_{k-1} B_{k}\right]=\alpha_{k}^{-1}\left(\mathrm{E}\left[B_{k}^{2}\right]+\sigma_{w}^{2}\right)-\alpha_{k} \sigma_{\varepsilon(k-1)}^{2} .
$$

Finally, plugging equations (13) and (10) in (11) and performing some algebraic manipulation one can show that

$$
\sigma_{\varepsilon(k)}^{2}=\frac{\alpha_{k}^{2} \sigma_{\varepsilon(k-1)}^{2}-\sigma_{w}^{2}}{\left.1+\left(A \mu_{X_{k}} \mu_{B_{k}}\left(1-\mu_{B_{k}}^{-2} \mathrm{E}\left[B_{k}^{2}\right]\right)+\left(\alpha_{k}^{2} \sigma_{\varepsilon(k-1)}^{2}-\sigma_{w}^{2}\right) \psi_{k}\right)\right) \mathbf{1}^{T} \boldsymbol{S}^{-1} \mathbf{1}} .
$$

Equations (10), (10) and (14) in conjunction with the system parameters completely determine the proposed filter. In the next section, we show that if an extra assumption on the input irradiance is made, the estimator is strongly simplified to a simple expression.

\section{Computer Implementation}

The computer implementation of the proposed filter depends basically upon the inversion of the matrix $\boldsymbol{S}$ in order to obtain the recursions for $\boldsymbol{K}_{k}$ and $\sigma_{\varepsilon(k)}^{2}$ (see equations (10) and (14) ). Recall that $\boldsymbol{S}=A^{2} \mathrm{E}\left[\boldsymbol{X}_{k} \boldsymbol{X}_{k}^{T}\right]+\sigma_{v}^{2} \boldsymbol{I}_{l_{k} \times l_{k}}-A^{2} \mu_{X_{k}}^{2} \mathbf{1 1}^{T}$. 
On one hand it is easy to realize that the matrix $\boldsymbol{M}=\sigma_{v}^{2} \boldsymbol{I}_{l_{k} \times l_{k}}-A^{2} \mu_{X_{k}}^{2} \mathbf{1 1}^{T}$ has diagonal elements $(m)_{i i}=\sigma_{v}^{2}-A^{2} \mu_{X_{k}}^{2}$ and off-diagonal elements $(m)_{i j}=$ $-A^{2} \mu_{X_{k}}^{2}, i \neq j$. On the other hand, the autocorrelation matrix $\boldsymbol{N}=\mathrm{E}\left[\boldsymbol{X}_{k} \boldsymbol{X}_{k}^{T}\right]$ has diagonal elements given by: $(n)_{i i}=\mathrm{E}\left[x_{k}(i) x_{k}(i)\right]=\mu_{X_{k}}^{2}+\sigma_{X_{k}}^{2}$, while the offdiagonal elements are: $(n)_{i j}=\mathrm{E}\left[x_{k}(i) x_{k}(j)\right]=\mu_{X_{k}}^{2}$. In addition, the assumption that $\boldsymbol{X}_{k}$ is uniformly distributed in a range $\left[X_{\text {min }}, X_{\text {max }}\right]$ implies that

$$
\begin{aligned}
\mu_{X_{k}} & =2^{-1}\left(X_{\max }+X_{\min }\right) \\
\sigma_{X_{k}}^{2} & =12^{-1}\left(X_{\max }-X_{\min }\right)^{2} .
\end{aligned}
$$

If we adjust a symmetric range for the input irradiance, say $\left[-X_{0}, X_{0}\right]$ for some $X_{0}>0$, this implies that $\mu_{X_{k}}=0$ and the matrix $S$ reduces to

$$
\boldsymbol{S}=\left(A^{2} \sigma_{X_{k}}^{2}+\sigma_{v}^{2}\right) \boldsymbol{I}_{l_{k} \times l_{k}}
$$

Therefore, no matrix inversion is required and equations (10) and (14) are simplified as well

$$
\begin{aligned}
\boldsymbol{K}_{k} & =K_{k} \mathbf{1}=\frac{\sigma_{\varepsilon(k)}^{2}}{A^{2} \sigma_{X_{k}}^{2}+\sigma_{v}^{2}} \mathbf{1}, \\
\sigma_{\varepsilon(k)}^{2} & =\frac{\alpha_{k}^{2} \sigma_{\varepsilon(k-1)}^{2}-\sigma_{w}^{2}}{1+l_{k}\left(\alpha_{k}^{2} \sigma_{\varepsilon(k-1)}^{2}-\sigma_{w}^{2}\right)\left(A^{2} \sigma_{X_{k}}^{2}+\sigma_{v}^{2}\right)^{-1}} .
\end{aligned}
$$

As a final consequence, the filter can be implemented by means of the following equation

$$
\hat{B}_{k}=\alpha_{k} \hat{B}_{k-1}+l_{k} K_{k}\left(\frac{1}{l_{k}} \sum_{i=1}^{l_{k}} y_{k}(i)-\hat{B}_{k-1}\right) .
$$

Remark: Assuming a symmetric range for $X$ implies that the offset NUC must be performed in the following way $\hat{x}_{k}(n)=A_{k}^{-1}\left(y(n)-\hat{B}_{k}\right)+x_{0}$, where the term $x_{0}$ adjusts the irradiance range $\left[-X_{0}, X_{0}\right]$ to the original range $\left[X_{\min }, X_{\max }\right]$.

\subsection{Initial Condition}

The initialization of the extended filter can be performed in the same way as is done in the case of the filter proposed in 11. This initialization process determines appropriate values for $\sigma_{\varepsilon(0)}^{2}, X_{\min }, X_{\max }, A, R_{B}(0)=\mathrm{E}\left[B_{0} B_{0}\right]$, and $\sigma_{v}^{2}$. The remaining parameters, namely $\alpha_{k}, l_{k}$, and $\sigma_{w}^{2}$, are addressed here. In order to obtain an expression for $\sigma_{w}^{2}$ we consider $W_{k}$ and the Gauss-Markov model (2) to obtain

$$
\mathrm{E}\left[B_{k} W_{k}\right]=\alpha \mathrm{E}\left[B_{k-1} W_{k}\right]+\mathrm{E}\left[W_{k} W_{k}\right]=\sigma_{w}^{2} .
$$

In addition we have that

$$
\begin{aligned}
\mathrm{E}\left[B_{k} B_{k}\right]-\alpha \mathrm{E}\left[B_{k-1} B_{k}\right] & =\mathrm{E}\left[B_{k} W_{k}\right] \\
R_{B}(0)-\alpha R_{B}(-1) & =\sigma_{w}^{2},
\end{aligned}
$$


so we can write $\sigma_{w}^{2}$ as

$$
\sigma_{w}^{2}=\left(1-\alpha^{2}\right) R_{B}(0)
$$

Finally, the initial value for the error variance is given by

$$
\sigma_{\varepsilon(0)}^{2}=\frac{R_{B}(0)\left(2 \alpha^{2}-1\right)}{1+l \frac{R_{B}(0)\left(2 \alpha^{2}-1\right)}{A^{2} \sigma_{X_{k}}^{2}+\sigma_{v}^{2}}} .
$$

\subsection{Convergence Analysis}

From equation (25) it is possible to obtain asymptotic results for $\sigma_{\varepsilon(0)}^{2}$ and $K_{k}$ when the length of each block, $l$, is long enough. Let us define the constant $a \triangleq R_{B}(0)\left(A^{2} \sigma_{X_{k}}^{2}+\sigma_{v}^{2}\right)^{-1}$ and firstly consider the cases when $k=\{0,1\}$

$$
\begin{aligned}
& \lim _{\alpha \rightarrow 1} \sigma_{\varepsilon(0)}^{2}=\lim _{\alpha \rightarrow 1} \frac{R_{B}(0)}{\left(2 \alpha^{2}-1\right)^{-1}+l a}=\frac{R_{B}(0)}{1+l a} \\
& \lim _{\alpha \rightarrow 1} \sigma_{\varepsilon(1)}^{2}=\lim _{\alpha \rightarrow 1} \frac{\alpha^{2} \sigma_{\varepsilon(0)}^{2}+\left(\alpha^{2}-1\right) R_{B}(0)}{1+l a\left(\alpha^{2} \sigma_{\varepsilon(0)}^{2}+\left(\alpha^{2}-1\right) R_{B}(0)\right)}=\frac{R_{B}(0)}{1+2 l a},
\end{aligned}
$$

Using mathematical induction one can show the following results

$$
\begin{aligned}
\lim _{\alpha \rightarrow 1} \sigma_{\varepsilon(k)}^{2} & =\frac{R_{B}(0)}{1+(k+1) l a}, \\
\lim _{\alpha \rightarrow 1} K_{k} & =\frac{a}{1+(k+1) l a} .
\end{aligned}
$$

Note that when $\alpha \rightarrow 1$, the variance of the terms $\sigma_{\varepsilon(k)}^{2}$ and $K_{k}$ are bounded (by zero) decreasing sequences parameterized by $l a$; hence, convergence is guaranteed. It must be remarked also that for $l=1$, equations (28) and (29) reduce to the equations found in [1].

For the situation when $\alpha \ll 1$, it is possible to demonstrate that the error variance is approximated by

$$
\sigma_{\varepsilon(k)}^{2}=M_{0} \cot \left(\frac{\pi^{2}}{2} \sqrt{a}\left(k+\theta_{0}\right) \arccos \alpha^{4}\right),
$$

where $M_{0}$ is selected such that (30) satisfies the relation: $\sigma_{\varepsilon(0)}^{2}=R_{B}(0)\left(\left(2 \alpha^{2}-\right.\right.$ $\left.1)^{-1}+l a\right)^{-1}$ and $\theta_{0}=(10 a \sqrt{l})^{-1}$. This relation is important because the equation (30) states that a wrong selection of $\alpha, l$ and $a$ parameters may result in divergent sequences for the filter parameters, and consequently, in a divergent NUC algorithm. Even more, $\sigma_{\varepsilon(k)}^{2}$ could turn out to be zero for any combination of the parameters satisfying

$$
k_{l}=\frac{1}{\pi \sqrt{a} \arccos \alpha^{4}}-\theta_{0},
$$

where $k_{l}$ is the last iteration of the algorithm when $\sigma_{\varepsilon\left(k_{l}\right)}^{2}=0$. Such a situation occurs for the frame $k_{l} l$. So, given a certain set of parameters, the quantity $k_{l}$ 
must be calculated in order to improve the convergence of the algorithm. For a desired large value of $k_{l}, \alpha$ must be very close to 1 , a needs to be small, and $l$ large. Finally, note that in equation (31) if $\alpha \rightarrow 1$ then $k_{l} \rightarrow \infty$, and in spite of that, no problem with the convergence is presented.

\section{Applications to Real IR Image Sequences}

The main goal of this section is to test the ability of the proposed method to reduce the $\mathrm{NU}$ in real $\mathrm{IR}$ video sequences. The sequences employed in this study have been collected using an $128 \times 128 \mathrm{InSb}$ FPA cooled camera (Amber Model AE-4128), operating in the $3-5 \mu \mathrm{m}$ range. In the set data, 3000 frames were collected at a rate of 30 frames per second, with a resolution of 16 bits per pixel.

Firstly, the initial conditions for the algorithm are calculated: The range for the input irradiance is defined to be [0,65535], i.e. the full dynamical range of the camera. Then the $\alpha$ and $l$ parameters can be obtained from [5]. If a short block of frames is employed, i.e. $l=100$ corresponding to approximate 3.33 seconds of data, and $\alpha$ is set to 0.95 it is possible to find $k_{l}$ using (31). If $a=0.1$, then at the frame $k_{l} l=153$ the variance of the error turns out to be $\sigma_{\varepsilon\left(l \times k_{l}\right)}^{2}=0$. If $a=1 \times 10^{-3}$ then $\sigma_{\varepsilon\left(k_{l} l\right)}^{2}=0$ for $k_{l} l=626$. On the other hand, if $\alpha=0.99995$ and $a=0.1$ then the product $k_{l} l=5023$ and in a such case the 3000 frames collected by the IR camera can be used to compensate for the NU noise.

Figures 1 (a) to (d) show, from left to right, an actual noisy frame and the corresponding corrected versions of such a frame when the NUC method proposed in this paper is employed using different set of parameters. A simple naked-eye inspection shows that the proposed method solely mitigates the NU noise when an adequate choose of the convergence parameters is made. The NUC performance is evaluated employing the so-called roughness index as well. (For details and definition see [4.) For such an index, the closer the value to zero the better the correction achieved. The roughness indexes for each case shown in Figure 1 are: (a) 2.5144, (b) 2.3489, (c) 2.2538, and (d) 2.2825. According to this index,

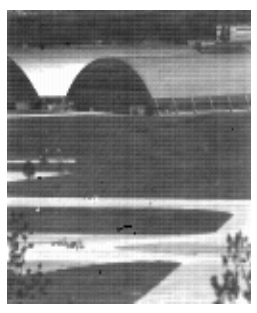

(a)

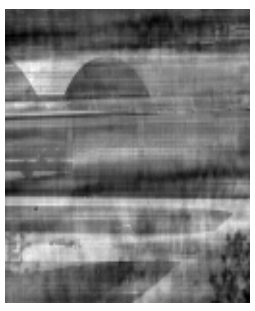

(b)

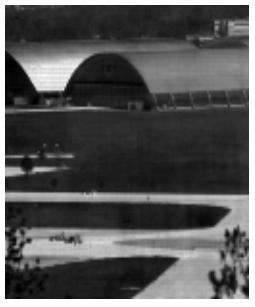

(c)

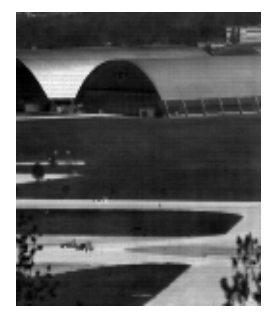

(d)

Fig. 1. A sample frame form a sequence of IR data taken at 1 PM: a) the corrupted raw frame. The corrected version of the raw frame obtained using the following parameters: b) $\alpha=0.95, a=0.1$, and $l=100$; c) $\alpha=0.99995, a=0.1$, and $l=100$; and d) $\alpha=0.99995, a=0.1$ and $l=300$. Visual inspection shows that a fairly good NUC is achieved the the last two sets of parameters. 
the set of parameters specified in the (c) and (d) cases are appropriated values for NUC.

\section{Conclusions}

In this paper an extended recursive filter for NUC in IR imaging system is presented. It was shown experimentally, using real IR data, that the method is capable to notably reduce the NU noise observed on the images. Indeed, the method has shown good reduction in offset $\mathrm{NU}$ when an adequate selection of the convergence parameters of the filter is made. Compared to 617, the main advantage of the method described here is its computational simplicity, which does not require any matrix inversion during the estimation process. Furthermore, the method generates a convergence parameter that is useful to stop the algorithm when the error variance is equal. In addition, no strong assumptions were made during the derivation of the algorithm. Namely, the assumption made are: (i) The input irradiance at each detector is a random variable uniformly distributed in a range common to all detectors; (ii) The input IR sequence of frames exhibits notorious offset NU, with slow temporal drift; and (iii) The drift in the offset NU is modeled by a discrete-time Gauss-Markov random process.

\section{References}

1. San Martin, C., Torres, S.: Statistical Recursive Filtering Estimation of Detector Offset Nonuniformity in Infrared Imaging System. In: Martínez-Trinidad, J.F., Carrasco Ochoa, J.A., Kittler, J. (eds.) CIARP 2006. LNCS, vol. 4225, pp. 464-473. Springer, Heidelberg (2006)

2. Scribner, D., Sarkady, K., Kruer, M.: Adaptive Nonuniformity Correction for Infrared Focal Plane Arrays using Neural Networks. Proc. of SPIE 1541, 100-109 (1991)

3. Scribner, D., Sarkady, K., Kruer, M.: Adaptive Retina-like Preprocessing for Imaging Detector Arrays. In: Proc. IEEE Int. Conf. on Neural Networks, vol. 3, pp. 19551960 (1993)

4. Torres, S., Vera, E., Reeves, R., Sobarzo, S.: Adaptive Scene-Based Nonuniformity Correction Method for Infrared Focal Plane Arrays. Proc. of SPIE 5076, 130-139 (2003)

5. Torres, S., Hayat, M.: Kalman Filtering for Adaptive Nonuniformity Correction in Infrared Focal Plane Arrays. The JOSA-A The Opt. Soc. of America 20, 470-480 (2003)

6. Torres, S., Pezoa, J., Hayat, M.: Scene-based Nonuniformity Correction for Focal Plane Arrays Using the Method of the Inverse Covariance Form. Applied Optics Inf. Proc. The Opt. Soc. of America 42, 5872-5881 (2003)

7. Pezoa, J., Hayat, M., Torres, S., Rahman, M.: Multimodel Kalman Filtering for Adaptive Nonuniformity Correction in Infrared Sensors. The JOSA-A The Opt. Soc. of America 23, 1282-1291 (2006)

8. Averbuch, A., Liron, G., Bobrovsky, B.: Scene Based Non-uniformity Correction in Thermal Images Using Kalman Filter. Elsevier Image and Vision Computing 25, 833-851 (2007) 